\section{Laser physics}

Laser Spectroscopy: Proceedings of the Second International Conference, Physics, Vol. 43). Edited by $S$. Haroche, J. C. Pebay-Peyroula, T. W. Hänsch and S. E. Harris. Pp. $x+468$. (Springer: Berlin and New York, 1975.) DM 45; $\$ 18.50$.

THE development of tunable laser systems has radically altered the nature of atomic and molecular physics. Used in conventional spectroscopy, the high intensity and monochromaticity of the tunable laser makes it an ideal probe of purely atomic properties such as excited state lifetimes, fine and hyperfine structure splittings and isotope shifts: When the laser is used in a dynamic sense, new and exciting non-linear, intensity-dependent features emerge. Finally the extremely short duration of pulses generated by pulsed modelocked lasers enables the photochemist to study ultrafast relaxation and transport mechanisms. This volume, demonstrates how laser spectroscopy has arrived at a semblance of maturity after a period of very rapid growth. The topics discussed here extend from the quantum electrodynamics of laser resonance fluorescence to laser isoMegève, June 1975. (Lecture Notes in the list of worthy results is endless. tope separation processes of economic importance. Invited papers presented at the conference are here printed directly from the authors' typescripts.

For me, some especially interesting papers were those of Cagnac on high resolution, two photon spectroscopy, Mollenauer on colour centre lasers, and Cohen-Tannoudji's review of the currently fashionable dynamic Stark effect in resonance fluorescence. Workers in laser physics or atomic physics will find much in this volume to entertain and to stimulate. One fascinating feature is the large number of papers, some with carefully spelled-out background histories and dates, devoted to laser isotope separation. On this the editors make the curious comment: "Unfortunately, the free flow of information was here still severely hampered by secrecy and classification, and the wisdom of including such a 'political' topic in the program has since been seriously questioned".

No doubt some of the new results presented at the conference for the first time will either appear or already have appeared elsewhere in the literature. But as a review of the status of the field, this volume serves a very useful purpose and can be recommended to workers in atomic and laser physics.

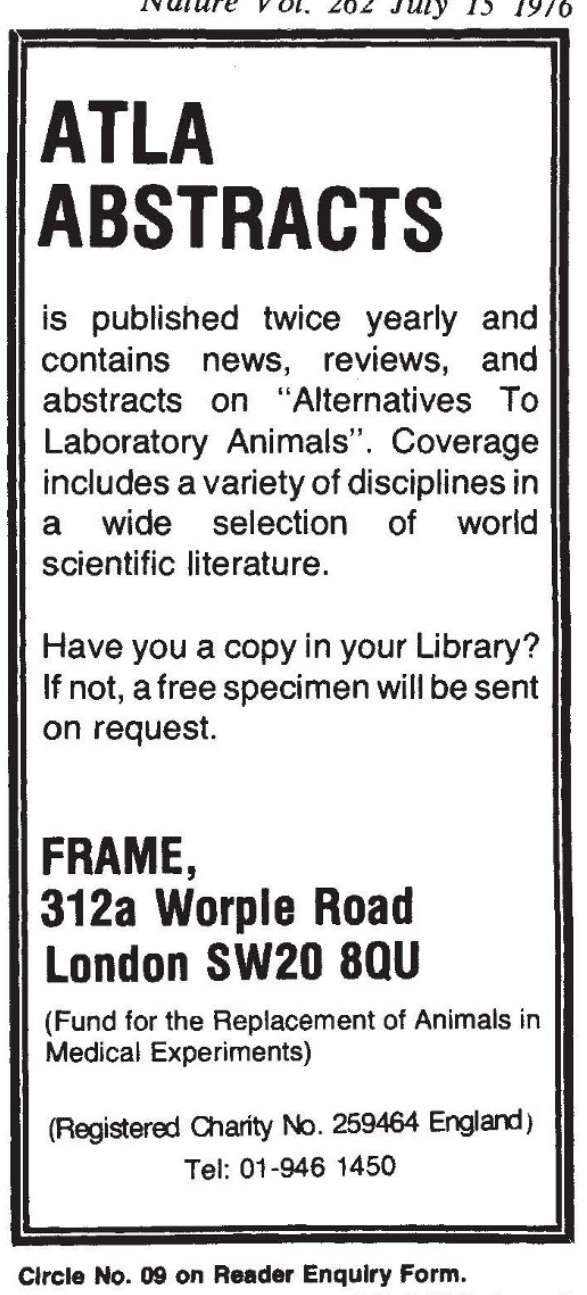

\section{Sound basis for plant virology course}

Plant Virology: The Principles. By Adrian Gibbs and Bryan Harrison. Pp. $x+292$. (Arnold: London, March 1976.) £16.

IN the Preface to this excellent book the authors describe its gestation period as 'lengthy', which is scarcely more informative than a piece of string; but as they state that they aimed to provide a text at the university teaching level, a need not filled by books which have since appeared, I suppose that this one grew from notes prepared for lectures given to students of the Imperial College, University of London, by Gibbs and Harrison some 10 years ago while they were both at Rothamsted. This speculation is relevant to an assessment, since a gestation period of several years would account for some characteristics which might otherwise puzzle or even irritate a reader. I am not suggesting that the book is out of date; far from it: I am referring to differences between chapters in style and depth. Problems of selection would have grown erratically as reference lists lengthened because research papers proliferated on fashionable topics; as the cost of publication rose at an accelerating rate, hard choices would have to be made.

The layout of the book provides a sound basis for a lecture course for undergraduates. After the history and scope of plant virology, some groups of viruses and the cryptogram system are described, followed by accounts of virus effects on plants, and methods of experimental transmission. The next chapter deals with the composition and structure of plant viruses in consideraable depth and seems over-elaborate compared with others. Purification and properties are well covered in chapter 6 , although the use of liquid nitrogen and of polyethylene glycol in extraction seem strange omissions. Assay methods separately considering infectivity, serology and physicochemical methods. After chapters on effects of inactivation and behaviour of viruses in plants, one on variation, strains and classification seems misplaced between them and those on means of transmission, ecology and ways of preventing crop losses. In my view, part of the penultimate and all of the final chapter should have been omitted and the space used to expand those chapters which seem unduly are clearly described in three chapters compressed-for example, 'Effect of Viruses on Plants'; the subjects in question are 'Viruses of Animals' and 'Plant Pathogens Confused With Viruses'.

The production is excellent (as befits its price), with clear type and illustrations and agreeably few typographical errors. I regret the absence of titles from the references, making it laborious to check that all are worth inclusion; one suspects that the authors' assurance that, in spite of their selection having been "somewhat arbitrary, most important papers are included," refers mainly to those by themselves and co-workers; otherwise the choice might best be described as whimsical.

The authors have disregarded their own warning in at least one instance. They rightly stress the hazards of identifying viruses on inadequate data, yet they apply the name "swollen shoot" to a virus of cacao trees in Trinidad. To the best of my knowledge, this and the West African swollen shoot virus have no established affinities besides a common vector.

Unquestionablv this book is a valuable addition to the few on plant viruses and will be welcomed esvecially by the small hand who teach the subject at English-spcaking universities

A. F. Posnette 